\title{
Stoichiometric molecular hydration of interstitial sites in a close-packed ionic lattice
}

\section{Chris Ling ${ }^{1}$, Frederick Marlton ${ }^{1}$, Alex Brown ${ }^{1}$, Andrey Maljuk², Bernd Büchner ${ }^{2}$, William Lewis ${ }^{1}$, Matthew Sale ${ }^{1}$, lan Luck ${ }^{1}$}

\author{
${ }^{I}$ The University of Sydney, Sydney, Australia; \\ ${ }^{2} I F W$-Dresden, Dresden, Germany; \\ chris.ling@sydney.edu.au
}

The hexagonal perovskite-type oxide $6 \mathrm{H}-\mathrm{Ba}_{4} \mathrm{Ta}_{2} \mathrm{O}_{9}$ undergoes an unconventional symmetry lowering lattice distortion when cooled below $1100 \mathrm{~K}$ in the presence of atmospheric water. This temperature corresponds to the onset of hydration, which reaches a stoichiometric value $6 \mathrm{H}-\mathrm{Ba}_{4} \mathrm{Ta}_{2} \mathrm{O}_{9}{ }_{1}^{1} / 2 \mathrm{H}_{2} \mathrm{O}$ by $\sim 500 \mathrm{~K}$. In the study to be presented here, we used a combination of diffraction, $a b$ initio calculations and spectroscopy to show that both processes are due to the incorporation of intact water molecules into the closepacked ionic lattice. The presence of very large $\mathrm{Ba}^{2+}$ cations in octahedral interstitial sites (perovskite $B$ sites) forces adjacent vacant octahedral interstitial sites to also expand, making room for occupation by water molecules, while also destabilizing the structure in a way that cannot be adequately addressed by conventional symmetry-lowering pathways on cooling. This gives rise to a synergistic hydration-distortion mechanism, which, to the best of our knowledge, is unique among close-packed ionic compounds. We will discuss the implications of our model for protonic and oxide ionic conductivity in hexagonal perovskites as fuel-cell membrane materials, and for earth sciences given the possibility that more examples could exist under high-temperature and pressure conditions.

Keywords: Hydration, ionic conduction, hexagonal perovskite, neutron diffraction, DFT 